\title{
Ética militar y estrategia. Una aproximación desde el realismo clásico y el neoliberalismo institucional ${ }^{1}$
}

https://doi.org/10.21830/9789585377127.07

\author{
Jesús Eduardo Moreno Peláez \\ Ximena Andrea Cujabante Villamiß \\ Humberto Librado Castillo ${ }^{4}$ \\ Escuela Superior de Guerra "General Rafael Reyes Prieto"
}

\section{Resumen}

El presente capítulo de libro analiza la relación entre la ética militar y la estrategia con base en dos teorías centrales de las relaciones internacionales: el realismo y el neoliberalismo institucional. Para tal fin, se utilizó una metodología cualitativa exploratoria, toda vez que son muy pocos los escritos que abordan esta temática, de tal manera que este capítulo constituye un aporte para futuras investigaciones sobre el tema. En este sentido, se discuten elementos esenciales de la definición de estrategia y ética militar, así como de

1 Este capítulo presenta los resultados colaborativos de dos proyectos de investigación: (1) "Ética militar en entornos complejos de seguridad y defensa: lecturas y aportes desde la experiencia de las Fuerzas Militares de Colombia”, del grupo de investigación Masa Crítica, de la Escuela Superior de Guerra "General Rafael Reyes Prieto", Colombia, categorizado en B por Minciencias y con código de registro COL0123247, y (2) "Mujeres de arma, seguridad y defensa nacional. Un análisis desde sus percepciones", del grupo de investigación en Ciencias Militares, de la Escuela Militar de Cadetes "General José María Córdova”, Colombia, categorizado en B por Minciencias y con código de registro COL0082556. Los puntos de vista pertenecen a los autores y no reflejan necesariamente los de las instituciones participantes.

2 Teniente Coronel (RA) del Ejército Nacional de Colombia. Profesional en Ciencias Militares de la Escuela Militar de Cadetes "General José María Córdova”. Magíster en Inteligencia Estratégica de la Escuela de Inteligencia y Contrainteligencia "Bg. Ricardo Charry Solano”. Magíster en Seguridad y Defensa Nacionales de la Escuela Superior de Guerra "General Rafael Reyes Prieto”. Orcid: https://orcid. org/0000-0001-8516-3893 - Contacto: jesus.moreno@esdegue.edu.co

$3 \mathrm{PhD}$ en Estudios Políticos y magíster en Asuntos Internacionales de la Universidad Externado de Colombia. Especialista en Negociación y Relaciones Internacionales de la Universidad de los Andes. Politóloga de la Universidad Javeriana. OrCID: https://orcid.org/0000-0002-5473-163X - Contacto: ximena. cujabante@unimilitar.edu.co

$4 \mathrm{PhD}$ (c) en Estudios Políticos y magíster en Análisis de Problemas Políticos Económicos e Internacionales de la Universidad Externado de Colombia. Politólogo de la Universidad Nacional de Colombia. Orcid: https:/orcid.org/0000-0001-8431-8611 - Contacto: humberto.librado@esdegue.edu.co 
su relevancia para la comprensión de los actores del sistema internacional y la forma en que estos elementos son abordados desde estas dos aproximaciones. Los resultados del estudio evidencian, a través de los principios expuestos por el realismo clásico, que hay una analogía entre el comportamiento estatal e individual, según la cual los seres humanos (los Estados) ansían el poder por encima de todas las cosas. Dichos valores que se consideran comunes a los individuos asociados en Estados y que se definen a través de principios morales, se proyectan a través de reglas, normas y convenciones que delimitan la forma de las conductas. Se concluye que el análisis desde el realismo clásico o el neoliberalismo institucional no solo constituye una fórmula para la comprensión académica, sino que además permite establecer marcos que condicionan el entendimiento de la ética y la estrategia militar.

Palabras clave: estrategia; ética militar; poder; realismo.

\section{Introducción}

Comprender la estrategia y sus componentes relacionados con la ética militar hace necesario establecer una articulación más amplia que permita ver cómo se problematiza esta interacción desde las teorías de las relaciones internacionales. En este sentido, los aportes de las visiones realistas y del neoliberalismo institucional permiten analizar la manera en que estos dos aspectos fundamentales se articulan en entornos complejos, lo cual llama la atención sobre todo a la hora de comprender la forma en que los actores involucrados en la política internacional llevan a cabo la operacionalización de la distinción ética.

La estrategia como aspecto central de la experiencia de las organizaciones estatales, tanto en su dimensión asociada a la seguridad y la defensa, como al marco más amplio de las labores administrativas, llama la atención sobre los límites de la racionalidad de los Estados. Esta discusión invoca entonces una dimensión humana que recuerda la necesidad de considerar las acciones estatales, entre ellas las militares, en un marco axiológico en el cual cotidianamente se lleva a cabo la decisión sobre los valores y, por ende, la distinción entre lo bueno y lo malo.

Desde esta perspectiva, reparar en las relaciones entre estas dos dimensiones, la estratégica y la ética militar, constituye un espacio de reflexión rele- 
vante para los actores involucrados en la actividad de los Estados, más aún si se considera que estas dimensiones estratégicas se establecen en relación con otros Estados que persiguen objetivos similares en el sistema internacional.

El presente capítulo de libro indaga de qué manera dos enfoques teóricos centrales en el estudio de las relaciones internacionales —el realismo y el neoliberalismo institucional — contribuyen a moldear la forma como comprendemos la relación entre la ética militar y la estrategia, que se traduce en prácticas concretas en los escenarios internacionales.

De este modo, la primera sección aborda de forma general las dimensiones de la estrategia y la ética; en la segunda, se analiza la manera en que el realismo ha comprendido las relaciones internacionales, así como su relación con la ética militar y la estrategia. Finalmente, en la tercera sección se estudia esta relación desde el neoliberalismo institucional a partir de los planteamientos de sus autores centrales.

\section{Estrategia militar}

La estrategia nace con el hombre y la necesidad de supervivencia y desarrollo. La palabra estrategia solo fue usada en los círculos castrenses desde finales del siglo XVIII. Específicamente, cuando Napoleón hablaba sobre esos temas se refería a la gran táctica. Por su parte, el archiduque austriaco Carlos planteó que la estrategia era la ciencia de la guerra y que ella esboza los planes y determina las operaciones militares (Solis, 2005, p. 18).

De hecho, el concepto de estrategia ha estado presente en todas las conducciones de las operaciones militares a lo largo de la historia de la humanidad. Si bien los principios de la estrategia solo se definieron con claridad en el siglo XIX, fueron aplicados en forma consciente e intuitiva por caudillos que supieron maniobrar sus fuerzas y emplear adecuadamente los medios y la geografía en las operaciones de guerra para lograr la victoria sobre el enemigo (Izcue et al., 2013).

En su concepción más básica, la estrategia es considerada como el arte de dirigir las operaciones militares para obtener la victoria sobre el adversario. La estrategia se ocupa del planeamiento y la dirección de campañas bélicas, así 
como del movimiento y disposición estratégica de las fuerzas militares con el propósito de vencer al enemigo (Izcue et al., 2013).

Para Clausewitz, la estrategia es el empleo del poder militar como herramienta para alcanzar el objetivo político (Izcue et al., 2013). De acuerdo con este autor, en la guerra existían solo dos actividades diferentes y con contornos definidos: "Preparar y conducir individualmente estos encuentros aislados y combinarlos unos con otros para alcanzar el objetivo de la guerra” (Solis, 2005, p. 18). La primera correspondía a la táctica, ya que se encarga de enseñar el uso de las fuerzas armadas en los encuentros; y la segunda era la estrategia, la cual se entendía como el uso de los encuentros para alcanzar el objetivo de la guerra (Solis, 2005, p. 18).

Es de anotar que el alcance de la guerra no solo es de índole militar, sino que implica todos los campos de la acción del Estado, de manera que se establecen los siguientes niveles: (1) la estrategia total, (2) las estrategias generales y (3) la táctica (Izcue et al., 2013).

La estrategia total está directamente a cargo del gobierno y es la encargada de concebir la dirección de la guerra total. Su función es definir la misión propia y la combinación de las diferentes estrategias generales: política, económica, diplomática y militar. En este orden de ideas, tiene a su cargo establecer la finalidad de la guerra, las políticas generales, las coordinaciones y las directrices para lograr que los diferentes dominios del Estado trabajen mancomunadamente para obtener su finalidad a través del conflicto bélico (Izcue et al., 2013).

Las estrategias generales, por su parte, se refieren a cada uno de los ámbitos subordinados. Una estrategia general (militar, política, económica o diplomática) tiene por función repartir y combinar las tareas de las acciones realizadas en las diferentes ramas de actividad del ámbito considerado. En este sentido, existe una estrategia general militar que combina el accionar de las diferentes fuerzas armadas al igual que las demás estrategias en los diferentes dominios del Estado (Izcue et al., 2013, p. 19).

Vale la pena mencionar que existe otro nivel de estrategia que emplean los comandantes operacionales durante las campañas en los teatros de operaciones, para lo cual usan fuerzas específicas (ejército, armada y fuerza aérea), 
fuerzas conjuntas o combinadas, las cuales corresponden al nivel operacional de la estrategia. Esta es la Estrategia Operacional, que está subordinada a la Estrategia Militar (Izcue et al., 2013, p. 20).

En este orden de ideas, la estrategia traza el plan de la guerra y para cumplir su propósito añade actos que conducirán a lograrlo. En este contexto surgen la estrategia terrestre, la marítima, la aérea, la estrategia conjunta y la pirámide estratégica. De acuerdo con Clausewitz, en lo que se refiere a la primera de estas, el ejército tiene por misión el aniquilamiento de las fuerzas organizadas adversarias en cooperación con las otras instituciones armadas, mediante el combate y la ocupación del territorio enemigo (Solis, 2005, p. 20). Asimismo, sostenía que los procedimientos de la guerra terrestre se basan en el logro de éxitos parciales encadenados entre sí hasta lograr el éxito final. En este orden de ideas, constituye una ampliación de la táctica. La estrategia marítima, por su parte, hasta la década de los setenta se preocupaba únicamente por su rol en los conflictos, pero hoy por hoy tiene concentrada sus funciones en la disuasión oceánica, el dominio del mar, la proyección de poder y la presencia (Solis, 2005, p. 22). En lo que se refiere a la estrategia aérea, esta tuvo una amplia aplicación en el desarrollo de la Segunda Guerra Mundial. Esta constituye la última de las estrategias independientes, ligadas a un medio geográfico particular. Las operaciones aéreas se preocupan por dos actividades: el control y la proyección.

Es de anotar que los alcances estratégicos de las operaciones aéreas no dependen de las destrucciones causadas al enemigo, sino del efecto favorable obtenido para las propias fuerzas (Solis, 2005, p. 23). En cuanto a la estrategia conjunta, esta se ha enmarcado en los avances tecnológicos que han impactado el campo militar con la fabricación de armamento teleguiado, el cual se caracteriza por su alta precisión y letalidad. De esta forma, las unidades de combate terrestre, navales y aéreas han ganado movilidad y capacidad para operar de día $\mathrm{y}$ de noche. Todos estos perfeccionamientos han contribuido a mejorar la interoperatividad de los medios y, por lo tanto, facilitado la conducción conjunta (Solis, 2005, p. 24).

Finalmente, la pirámide estratégica se refiere a las guerras actuales, en las cuales se forma una pirámide político-estratégica. En la punta se ubica la 
política, que irradia la estrategia total que tiene como propósito lograr la paz. Como parte de esta estrategia total, se encuentra la estrategia militar direccionada a ganar la guerra neutralizando o destruyendo las fuerzas enemigas (Solis, 2005, p. 25). En el peldaño inferior se ubica la estrategia conjunta, que tiene la finalidad de lograr el dominio del espacio de la batalla con la participación de las estrategias terrestre, marítima y aérea, para lo cual potencia cada una de estas estrategias (Solis, 2005, p. 25).

\section{Ética versus moral}

Con el propósito de ahondar en el concepto de la ética militar desde la perspectiva de la teoría realista de las relaciones internacionales, se considera importante iniciar definiendo la ética y la moral, ya que, aunque son dos conceptos diferentes, socialmente son utilizados indistintamente como si significaran lo mismo. No obstante, resulta imprescindible distinguir con precisión la definición de cada uno de estas términos.

En este sentido, la ética se refiere a un ámbito reflexivo y teórico donde se razona sobre lo bueno y lo malo en las acciones humanas que realiza el individuo. La ética considera los actos libres del ser humano y les otorga una valoración, argumentos y razones para concluir que son adecuados y valiosos (Moliner, 2018b, p. 6). La moral, por su parte, se conforma a través de los principios que a partir de la reflexión ética definen y marcan el conjunto de acciones concretas del individuo. En este orden de ideas, la moral representa el ámbito práctico y es donde se orienta la conducta del ser humano. Frecuentemente, se apoya en un conjunto de reglas, principios, valores e ideales que establecen el comportamiento humano (Moliner, 2018b, p. 6).

Asimismo, es importante abordar la diferencia entre la moral y el derecho. Dicha distinción resulta importante para los militares por el papel que ejerce el Derecho Internacional Humanitario en sus funciones y cometidos, así como por la reflexión ética a la moral militar (Moliner, 2018a, p. 17). 


\section{La ética militar: una aproximación desde el realismo clásico}

En este orden de ideas, y una vez definidos los términos de ética y moral, a continuación se abordan los planteamientos que desde las relaciones internacionales intentan explicar la dinámica de la política internacional a la luz de la teoría del realismo clásico.

El nacimiento, desarrollo y consolidación de la disciplina de las relaciones internacionales han estado asociados con los hechos globales, especialmente aquellos ligados a los Estados Unidos y su posicionamiento en el entorno mundial. En palabras de Brian Schmidt (2002), el poder de Estados Unidos en el contexto externo de la posguerra, así como su posicionamiento político y económico a través de las alianzas durante la Guerra Fría, fueron decisivos para comprender el desarrollo que ha tenido el campo de las relaciones internacionales.

El estudio histórico convencional del campo de las relaciones internacionales ha buscado dar cuenta de su desarrollo a partir de dos supuestos historiográficos. Uno de estos hace referencia a que la historia de la disciplina se puede explicar en términos de una tradición clásica, de la cual los académicos modernos son herederos, mientras que el segundo supuesto es que los eventos que ocurren en el escenario internacional han estructurado el desarrollo de las relaciones internacionales como disciplina (Schmidt, 1998, p. 15).

En cuanto al primer supuesto, existe una tendencia a explicar la historia de la disciplina a partir de una serie de fases sucesivas o, en términos de Kuhn, paradigmas. El propósito de utilizar marcos analíticos de referencia tomados de la filosofía y la historia de la ciencia ha sido demostrar los avances científicos y el progreso en el campo de estudio (Ferguson \& Mansbach, 1993, p. 22). En este sentido, la historiografía ortodoxa de las relaciones internacionales ha organizado la historia del desarrollo de la disciplina a través de los "grandes debates" (Schmidt, 2002, p. 10).

Hedley Bull es uno de los autores que plantean que la historia de la disciplina se puede explicar a partir de tres periodos sucesivos de actividad teórica: idealista (1920-1930), realista (finales de 1930 y 1940) y científico-social 
(finales 1950 y 1960) (Bull, 1972). C. R. Mitchel argumenta que es posible distinguir tres grandes escuelas de pensamiento dentro del debate general acerca de las bases de las ciencias sociales y en particular de las relaciones internacionales: la clásica, la behavioral (o científica) y la posbehavioral (o paradigmática) (Mitchel, 1980).

Miles Kahler ha denominado el primer debate como "el mito fundacional de la disciplina”, el cual tuvo lugar entre los idealistas y los realistas. Este debate dejó como resultado la reorientación del campo de las relaciones internacionales hacia una dirección más práctica y científica. Los idealistas eran reconocidos como un grupo de pacifistas utópicos y legalistas que enfocaban más su atención en reformar las políticas internacionales que en analizar la realidad de las políticas entre las naciones. El debate tuvo lugar cuando el sistema de la Liga de las Naciones falló y devino la Segunda Guerra Mundial. Esto acarreó una severa crisis para el paradigma idealista, el cual fue reemplazado por el realismo, que se destacaba por su habilidad para explicar racionalmente el persistente conflicto por el poder entre las naciones (Kahler, 1997). La historia de este debate continúa ejerciendo una fuerte influencia en la forma como la disciplina entiende su propia historia (Schmidt, 2002, p. 11).

$\mathrm{Y}$ es justamente en el marco de este debate que Hans Morgenthau propone los planteamientos del realismo político, los cuales han permanecido en el tiempo y aún continúan siendo tan vigentes como en ese entonces. A continuación, se intenta abordar la ética y la estrategia militar a la luz de los postulados realistas y su aplicabilidad al contexto global actual.

Morgenthau (1948, p. 12) plantea seis principios básicos para comprender la aplicabilidad y funcionalidad del realismo político en la política internacional. En este sentido, estos principios ofrecen las claves para poder comprender los postulados realistas. El primero intenta realizar una analogía entre la política y la sociedad al plantear que esta obedece a leyes objetivas que se arraigan en la naturaleza humana (Morgenthau, 1948, p. 12). El segundo principio, por su parte, plantea que el elemento que permite al realismo encontrar su rumbo en el panorama de la política internacional es el concepto de interés definido en términos de poder. Este proporciona el enlace entre la razón y los hechos. Para Morgenthau, los buenos motivos se convierten en seguridad 
contra las políticas deliberadamente malas, sin garantizar la moral o el éxito político de las políticas que inspiran. En este sentido, la ética juzga, de manera abstracta, la calidad moral de los motivos, mientras que la teoría política juzga la voluntad y la acción del estadista (Morgenthau, 1948, p. 14).

En cuanto al tercer principio, este hace referencia a que el interés definido en términos de poder es una categoría objetiva que constituye la esencia de la política internacional (Morgenthau, 1948, p. 19). En este sentido, el tipo de interés es determinante de las acciones políticas en un periodo específico dependiendo del contexto político y cultural dentro del cual se formula la política exterior. Los objetivos que pueden perseguir los Estados son diversos e incluyen distintos temas (Morgenthau, 1948, p. 20).

El cuarto principio hace referencia a que el realismo político conoce el significado moral de la acción política. En este sentido, se tiene conciencia de la inevitable tensión entre los preceptos morales y los requerimientos de una acción política exitosa (Morgenthau, 1948, p. 21). En este orden de ideas, sostiene que los principios morales universales no pueden aplicarse a los actos de los Estados en una formulación abstracta y universal, sino filtrados a través de circunstancias concretas de tiempo y lugar (Morgenthau, 1948, p. 21).

Continuando con la moralidad, el quinto principio se niega a identificar las aspiraciones morales de una nación particular con los preceptos morales que gobiernan el sistema internacional. El hecho de que el interés sea concebido en términos de poder permite estar un poco separados de los excesos morales (Morgenthau, 1948, p. 22). Finalmente, el sexto principio plantea la profunda diferencia entre el realismo político y las diferentes escuelas de pensamiento, así como la consecuente autonomía del realismo político en la esfera política, ya que este piensa el interés en términos de poder (Morgenthau, 1948, p. 23).

Como se puede apreciar a través de los principios expuestos por el realismo clásico, se hace una analogía entre el comportamiento estatal y el individuo. En este sentido, sostiene que los seres humanos (los Estados) ansían el poder por encima de todas las cosas. De hecho, algunas de las críticas a esta teoría justamente sostienen la falta de un concepto moral. Sin embargo, diferentes autores realistas han argumentado que el realismo clásico no asume que la moral está ausente de las relaciones internacionales, sino que considera que 
toda acción basada en la moral es contraproducente a los intereses del Estado, principalmente a su seguridad y supervivencia (Clulow, s. f.).

Vale la pena traer a colación, como se mencionó anteriormente, que la teoría centra su análisis en el Estado. Este Estado se caracteriza por ser unitario y racional. Unitario por cuanto es una unidad política soberana sobre su propio territorio y racional porque los realistas asumen que el Estado siempre adoptará la decisión más eficiente, dados los recursos y las capacidades disponibles con miras a garantizar su seguridad y lograr el poder. Es así como, para el realismo, las relaciones internacionales son un perpetuo proceso de negociación sobre la conquista y la redistribución de recursos y bienes escasos (Clulow, s. f.). En este entorno, en un mundo entre iguales (Estados soberanos) los poderosos tienen mayores oportunidades sobre los débiles, de manera que la capacidad militar adquiere una importancia relevante en el juego de la política internacional.

\section{El realismo clásico, la ética militar y la estrategia}

Desde la ética militar, Frowe (2011) sostiene que las referencias a las reglas de la guerra deberían ser entendidas como referencias a las reglas morales de la guerra como son estudiadas desde el enfoque de la guerra justa. En este sentido, la necesidad de la ética militar radica en que permite llegar mediante la razón y el juicio moral a que el ejercicio de la guerra debe estar justificado en razones éticas (Moliner, 2018a). Otra cuestión ética que se plantea es la necesidad y universalidad de los juicios morales, la consideración de si esos principios y valores que sustentan los códigos éticos varían de unos pueblos y culturas a otros. En este sentido, si la ética universal facilita la supervivencia humana, entonces no cabría plantearse éticas culturales (Moliner, 2018a, p. 8), planteamientos que se encuentran inmersos en los postulados realistas de la política internacional.

Retomando nuevamente el realismo clásico, este marco teórico plantea la importancia de la capacidad militar de los Estados. En este orden de ideas, los actores estatales deben procurar que se fomente el fortalecimiento militar como sinónimo de poder en la escena internacional. En este sentido, a la hora de intentar relacionar el realismo clásico y la ética militar resulta de gran rele- 
vancia tener en cuenta en el siglo XXI las implicaciones éticas que aparecen con el desarrollo y la utilización de nuevas tecnologías en el campo de batalla (Moliner, 2018b). Al respecto, se debe anotar que las nuevas tecnologías siempre se han incorporado progresivamente en las guerras y han modificado su conducción. No obstante, en otras ocasiones son las guerras las que han promovido los avances científicos y, de este modo, han transformado las estructuras militares, la gestión de los conflictos y las responsabilidades de los militares. Estos cambios también modifican el comportamiento de los soldados en el combate, pues les imponen conductas y actitudes que en otros momentos se enmarcarían como actos de valor heroico, elemento fundamental del ethos militar (Moliner, 2018b, p. 22).

Teniendo en cuenta que, desde la óptica del realismo clásico, la política internacional es motivada por el interés definido en términos de poder, en el ámbito de la ética militar el uso de la fuerza militar como un elemento en la política del Estado se enmarca dentro del ius ad bellum. En este orden de ideas, el principio de causa justa, y por extensión de guerra justa, es relevante en los nuevos desarrollos que presentan los conflictos bélicos. En el campo del ius in bello, vale la pena mencionar los problemas éticos relacionados con el avance de la ciencia y la tecnología (Moliner, 2018b, p. 28).

$\mathrm{Al}$ respecto, Moliner (2020) plantea que no es correcto hablar del concepto de ética militar como universal en el tiempo y en el espacio, ya que hay una ética militar hoy y ahora de acuerdo con el contexto. Es decir, lo que en un momento determinado es éticamente válido para una colectividad, no necesariamente será aceptado en otro momento histórico, ya que entran a jugar factores como los avances tecnológicos. No obstante, hay autores que sostienen que la ética militar existe sin fecha ni lugar, en la medida en que no hay alteración en la naturaleza propia de la función militar, razón por la cual consideran que la ética militar es un patrón constante (Moliner, 2020, p. 13).

De acuerdo con los conceptos de ética militar y estrategia vistos a la luz de la teoría realista de las relaciones internacionales, se puede entrever que el Estado — como actor racional que busca lograr su interés definido en términos de poder en un sistema internacional caracterizado por la anarquía y su subsecuente incertidumbre — pondrá a su alcance la estrategia militar que le permita 
lograr sus intereses en el escenario mundial, en donde la capacidad militar se convierte en un referente de poder. De esta forma, las diferentes estrategias (terrestre, marítima, aérea y conjunta) se convierten en herramientas para que el Estado logre su cometido. Finalmente, la pirámide estratégica refleja cómo los intereses políticos del Estado dentro de la política mundial irradian tanto la estrategia como la ética militar.

\section{Algunas nociones del neoliberalismo institucional}

Para el caso del neoliberalismo institucional, vale la pena avanzar en unas consideraciones generales que recogen las ideas de esta teoría sobre las condiciones del sistema internacional y las posibilidades de cooperación en entornos de anarquía, aunque no de caos, caracterizados por una creciente institucionalización frente a intereses compartidos, que delimitan valores comunes entre las naciones y aportan una dimensión ética de las relaciones internacionales y, a su vez, de los Estados que actúan en él y desde luego de los profesionales militares, quienes tienen un papel preponderante en el sistema internacional.

Un primer aspecto relacionado con la visión del neoliberalismo institucional se refiere a las características del orden internacional. No se trata de una visión de carácter hobbesiana del sistema internacional, en donde cada una de las autoridades tiene sus armas apuntadas listas para los enfrentamientos por la desconfianza y el recelo de las actuaciones de los demás, imagen asociada tradicionalmente a la idea de estado de guerra. Por el contrario, su interpretación demuestra que a pesar de la inexistencia de autoridades absolutas sobre todo el sistema y de que la relaciones formales de dominio y subordinación no resultan claras, tampoco se trata de un espacio de caos perpetuo. En palabras de Waltz: "El gobierno mundial, si bien no se puede confiar en que sea pacífico, no llega a ser un caos sin remedio" (Waltz, 1997, p. 915).

El sistema internacional no tiene entonces las mismas características en el mundo contemporáneo que el que ofrecía el sistema europeo tras los acuerdos de Westfalia en el siglo XVII.

En este sentido, la visión neoliberal institucionalista reconoce que la política mundial, si bien no está organizada formalmente, no carece totalmente 
de instituciones y procedimientos ordenados (Waltz, 1979, p. 114). De esta manera, estos marcos institucionales se comprenden como un conjunto de patrones recurrentes que moldean los comportamientos de los actores, en este caso los actores del sistema internacional.

Un segundo aspecto relevante en su interpretación del sistema internacional se encuentra en la institucionalización y la descentralización. La institucionalización hace referencia a la manera en que el comportamiento de los actores se reconoce como reflejo de reglas, normas y convenciones que interpreta para decidir de esta manera su actuación en este plano. Sobre este tipo de aspectos, se encuentran ejemplos como el reconocimiento de las misiones diplomáticas, la visión de la soberanía territorial, memorandos de entendimientos internacionales, elaboración de agendas multilaterales, movimiento de tropas y declaratorias de guerra internacional, todos aspectos en los cuales existe una serie de convenciones vinculadas con dichas acciones (Keohane, 1993).

Cabe señalar que estos grados de institucionalización ejercen un efecto significativo en el comportamiento de los gobiernos desde la perspectiva de los Estados y que los alcances en la forma en que constriñen sus acciones son variadas, sin menospreciar los elementos relacionados con la riqueza y el poder. Una síntesis de los aspectos en los que estas dimensiones inciden en el comportamiento de los actores del sistema internacional señala que sus efectos son perceptibles en (1) el conocimiento de la información del sistema y las oportunidades para la negociación, (2) la capacidad de los gobiernos para hacer valer las convenciones y practicar sus propios compromisos, y (3) la expectativa sobre el acuerdo (Keohane, 1993).

Desde estos elementos, la visión del neoliberalismo institucional —en el marco de las dificultades para la cooperación en el sistema internacionalreconoce la potencialidad de los humanos y del sistema internacional de los Estados para comunicarse y entablar acuerdos, reconociendo que estos acuerdos varían en la historia dependiendo del tipo de políticas y el reconocimiento de su carácter vinculante, que los hace rutinariamente obedecidos (Aggarwal, 1985, p. 31). 
Un tercer aspecto de la visión del neoliberalismo institucional se encuentra en la centralidad del Estado en la política mundial. En este caso, pese al reconocimiento de la emergencia de nuevos actores en el sistema internacional, priman las actuaciones de estos Estados, toda vez que son ellos los que se comportan en el marco de reglas formales e informales y motivan de este modo cambios de la arquitectura internacional, tanto como de su orden interior. En este sentido, este actor clave que es el Estado provee las fuerzas materiales de la política mundial (Keohane, 1993).

En este escenario, el neoliberalismo institucional asume la existencia de instituciones internacionales que pueden contar con las siguientes formas: en primer lugar, organizaciones intergubernamentales formales, capaces de controlar la actividad internacional y que están deliberadamente establecidas y diseñadas por los Estados. En segundo lugar, los llamados regímenes internacionales, es decir, instituciones con reglas explícitas en las que coinciden los gobiernos, por ejemplos la Ley del Mar y el régimen monetario internacional. Por último, se señala las convenciones, que son instituciones informales con entendimientos implícitos, que permiten construir escenarios de coordinación, por ejemplo en temas como la inmunidad diplomática antes de su codificación normativa (Young, 1983).

\section{Neoliberalismo institucional, implicaciones sobre la ética militar y la estrategia}

A partir de estos elementos de la visión del neoliberalismo institucional, para comprender su relación con la ética militar resulta importante observar de qué manera se relacionan estos planteamientos con las dimensiones de la ética militar señaladas previamente frente al ius ad bellum, ius in bellum y el ius post bellum. Luego se hará una revisión general de la visión ético-normativa de la perspectiva en general.

De acuerdo con el contenido de este artículo, plantear la forma en que se relacionan críticamente la ética militar y la estrategia requiere una aproximación en la que se considere esta relación en la perspectiva de los Estados y en un marco más amplio desde las distintas teorías de las relaciones internacionales. 
La consideración sobre la dimensión ética refleja el interés de los Estados de mantener uno de sus aspectos centrales, el monopolio de la coacción física, en el marco de la legitimidad necesaria para continuar con su ejercicio. En virtud de esta dimensión, se considera que la ética refiere los contenidos de las democracias representativas contemporáneas y deduce de ella los valores sociales necesarios para la vida social. Dichos valores que se consideran comunes a los individuos asociados en Estados y que se definen a través de principios morales, se proyectan a través de reglas, normas y convenciones que delimitan la forma de las conductas. Desde luego, para el caso de la ética militar, esta versa sobre dimensiones complejas al lidiar con el aspecto propio de la violencia estatal y de la guerra, en el entendido de mantener el equilibrio entre la disuasión y el carácter oportuno de su uso socialmente aceptable (Moliner, 2018c). Vista desde una perspectiva amplia,

la ética considera los actos libres del ser humano y les otorga una valoración, argumentos y razones concluyendo que son adecuados y valiosos por oposición a otros que son malos e inapropiados. O sea, se ocupa de discernir y definir la bondad y la maldad de los actos humanos, de definir y justificar los valores y las normas de conducta y de determinar la especificidad de la conducta moral frente a otros tipos de conducta. (Moliner, 2020, p. 77)

Y en el sentido específico de lo militar, versa sobre los

cimientos morales de la profesión de las armas, los valores que constituyen su núcleo, los principios que guían a los que ejercen esa profesión y las responsabilidades esenciales que deben asumir en la defensa y protección de su país. Se refiere a lo que hacen y cómo lo desarrollan, pero también a lo que se espera de su comportamiento, a sus relaciones profesionales e incluso a la percepción que tienen de ellos sus conciudadanos. (Moliner, 2020, p. 77)

Son múltiples los retos que determinan el entendimiento de esta ética en su carácter comprensivo sobre la guerra, tanto en el ius ad bellum, ius in bellum y el ius post bellum. A continuación, se presentan los elementos relacionados.

En lo que concierne al ius ad bellum, existe una serie de asuntos que implican una discusión sobre la ética militar. Al respecto, se pueden señalar aspectos como el uso de la fuerza militar en la política del Estado para alcanzar la paz; la relevancia del principio de causa justa y guerra justa; la discusión 
sobre la validez del principio de legítima defensa y, en particular, de defensa preventiva; el principio de lealtad al Estado democrático y la justicia de la guerra en la que interviene; la presencia de actores no estatales en el marco de los conflictos, la responsabilidad de proteger y la salvaguarda de los Derechos Humanos (Moliner, 2020).

En esta dimensión, es visible la relación con la visión del neoliberalismo institucional, en la medida en que muchos de estos temas se establecen en el marco de las organizaciones internacionales y de los regímenes internacionales. $\mathrm{Al}$ respecto, es notable el papel que tienen las organizaciones intergubernamentales como foro para las tensiones internacionales referidas a los temas bélicos. A su vez, en el marco de estas organizaciones y de las voluntades de los Estados, se han constituido convenciones y protocolos internacionales que determinan ciertas valoraciones que luego son incorporadas por los Estados y que se constituyen en marco de las interpretaciones éticas de los militares. Aspectos asociados, por ejemplo, a los Derechos Humanos y su protección en todos los escenarios muestran el carácter relevante de esta observación y la incidencia en la práctica.

A su vez, esto tiene una implicación de carácter estratégico, si se considera que los planteamientos estratégicos de los Estados deben tener en cuenta la observancia de estos preceptos, que se deben acatar en la medida en que puedan ser objeto de sanciones en el sistema internacional.

En el caso del ius in bellum, se encuentran elementos como la aplicación de los principios de uso apropiado de la fuerza militar y evitar los daños colaterales en un escenario de crecimiento tecnológico y de nuevos entornos de la guerra, ahora asociados a escenarios urbanos con mayor presencia de civiles. Otros aspectos están asociados a elementos de la ciberguerra y la posibilidad de asegurar la justicia y la rendición de cuentas; los modos de combate en acciones de contrainsurgencia y su relación con la erosión del principio de inmunidad, y la presencia cada vez mayor de civiles no combatientes en los entornos militares (Moliner, 2020).

Desde esta perspectiva, es evidente que la visión del neoliberalismo institucional prioriza la capacidad de los acuerdos. Cabe anotar que muchos de estos aspectos se encuentran en desarrollo a través de la participación de los Estados y muestran que hay esfuerzos en materia de construir espacios razonables para los convenios. No obstante, dada la novedad de algunas de 
estas cuestiones, la velocidad con la que se construyen dichas instituciones en el sistema internacional no permite en muchas ocasiones dar una respuesta pertinente para las comunidades. Un aspecto visible de esta dimensión está relacionado con la regulación de las compañías militares privadas y su participación en conflictos bélicos y en las guerras (Urueña, 2019).

Por su parte, las dimensiones de la valoración ética en torno al ius post bellum se remiten a los deberes con el vencido y las medidas aplicables, así como al compromiso de avanzar hacia una paz duradera que tenga en cuenta el respeto por los factores sociales y culturales propios de las comunidades (Moliner, 2020).

En la visión neoliberalista institucional, este aspecto adquiere mucha relevancia en el reconocimiento de la capacidad institucional para mediar en el contexto posterior a la guerra. Por lo tanto, se reconoce la potencialidad de la cooperación y la coordinación en acciones relacionadas con los procesos de construcción del Estado, así como en las consideraciones generales de los cambios de regímenes y los efectos de las transiciones económicas y políticas.

En un marco más amplio, se encuentra una crítica a la exposición del carácter ético propuesto desde la perspectiva del neoliberalismo institucional como se ha establecido hasta aquí. Esta aproximación parte de una premisa que no problematiza la capacidad normativa del enfoque, de manera que muchas veces en el marco de las necesidades de comprensión prevalecen connotaciones positivas de paz, seguridad y orden que transmiten igualmente juicios de valor.

Elementos asociados, por ejemplo, a la cooperación y la coordinación no permiten evidenciar la asimetría de los actores que participan en el sistema internacional. La visión sobre esta cooperación y su capacidad transformativa muestra que no se comprende que dichos proyectos pueden conducir a situaciones de mayor inequidad, si se considera que hay actores que son capaces de imponer la agenda a otros países y determinar desde allí prácticas que no necesariamente benefician a todos los participantes o se vinculan a las instituciones creadas en el sistema internacional (Richardson, 2008).

En términos generales, la aproximación en el neoliberalismo institucional parte de un principio utilitarista que considera a los actores estatales desde la perspectiva del individualismo metodológico, en el cual los actores maximizan sus beneficios en el sistema internacional, sin tener en cuenta que 
la asociatividad internacional asume costos diferentes para los participantes (Richardson, 2008).

Esta crítica general también llama la atención sobre la discusión de la estrategia y su relación con la ética militar. Específicamente, en la práctica sobrevienen discusiones sobre si la participación o no en una determinada operación o en una guerra contribuye al bienestar de los Estados y al cumplimiento de sus objetivos democráticos.

\section{Conclusiones}

El recuento que se ha presentado en el presente capítulo permite observar el modo en que cada una de las teorías aborda la relación entre estrategia y geopolítica. Plantear desde una perspectiva amplia la dimensión de la estrategia y la ética militar permite problematizar su relación con la actuación práctica de los Estados y los actores en general relacionados con los procesos de toma de decisión.

Estos procesos que lidian con la relación entre la estrategia y la geopolítica pueden ser revisados desde enfoques diferentes, como el realismo y el neoliberalismo institucional, los cuales se han revisado en este capítulo y se han problematizado en torno a la ética militar y la estrategia.

La revisión de estos elementos permite observar que el análisis desde uno $\mathrm{u}$ otro enfoque no solo constituye una fórmula para la comprensión académica, sino que además permite establecer marcos que condicionan el entendimiento de la ética y la estrategia militar. En este sentido, las teorías presentan cargas valorativas en sí mismas que condicionan la discusión sobre estas dimensiones y que es importante considerar tanto en el cuestionamiento sobre la objetividad teórica como en sus consecuencias prácticas.

\section{Referencias}

Aggarwal, V. (1985). Liberal protectionism: The international polities of Organized Textile Trade. University of California Press.

Bull, H. (1972). The theory of international politics 1919-1969. En B. Porter, The Aberystwyth paper: International politics 1919-1969. Oxford University Press 
Clulow, G. (s. f.). Una visión introductoria a los principios del realismo político. https://revistas. ort.edu.uy/letras-internacionales/article/view/338/339

Frowe, H. (2011). The ethics of war and peace. London Routledge.

Izcue, C., Arriaran, A., \& Tolmos, Y. (2013). Apuntes de estrategia operacional. División de Publicaciones de la Escuela Superior de Guerra Naval.

Kahler, M. (1997). Inventing international relations: International relations after 1945. En M. Doyle \& J. Ikenberry (eds.), New thinking in international relations theory (pp. 20-53). Westview.

Keohane, R. (1993). Institucionalismo neoliberal: Una perspectiva de la política mundial. En Instituciones internacionales y poder estatal (pp. 14-37). Grupo Editorial Latinoamericano.

Mitchel, C. R. (1980). Analysing the "Great Debates": Teaching metodology in a decade of change. En R. C. Kent \& G. P. Nielsson (eds.), The study and teaching of international relations. Frances Pinter Publisher.

Moliner, J. (2018a). ¿Por qué es importante la ética militar? Revista Española de Defensa. http://www.ieee.es/Galerias/fichero/panoramas/Panorama_geopolitico_2014.pdf

Moliner, J. (2018b). ¿Qué es la ética militar? http://www.ieee.es/Galerias/fichero/docs_ marco/2018/DIEEEM16-2018_EticaMilitar_JAMoliner.pdf

Moliner, J. (2018c). ¿Qué es la ética militar? Boletín IEEE, 11, 615-643.

Moliner, J. (2020). La ética militar y su importancia para el militar profesional. http://e-spacio. uned.es/fez/eserv/tesisuned:ED-Pg-SegInt-Jamoliner/MOLINER_GONZALEZ_ JUAN_ANTONIO_Tesis.pdf

Morgenthau, H. (1948). Politica entre las naciones. La lucha por el poder y la paz. GEL.

Richardson, J. L. (2008). The ethics of neoliberal institutionalism. The Oxford Handbook of International Relations (pp. 222-232). Oxford University Press.

Schmidt, B. (1998). The political discourse of anarchy. State University of New York Press.

Schmidt, B. (2002). On the history and historiography of international relations. En W. Carlsnaes, T. Risse \& B. Simmons (eds.), Handbook of international relations (pp. 3-22). Sage.

Solis, E. (2005). Evolución de la estrategia militar. Revismar, (1), 17-25. https://revistamarina. $\mathrm{cl} /$ revistas/2005/1/solis.pdf

Urueña-Sánchez, M. I. (2019). Las compañías militares de seguridad privada: ¿Los nuevos mercenarios? Criminalidad, 61(1), 97-110.

Waltz, K. N. (1979). Theory of international polities. Addison-Wesley.

Waltz, K. N. (1997). Evaluating theories. American Political Science Review, 91(4), 913-917.

Young, O. (1983). Regime dynamics: The rise and fall of international regimes. En S. Krasner (comp.), International regimes (pp. 93-114). Cornell University Press. 